Paper

A4

July 2002

\title{
The one-loop effective action for quantum electrodynamics on noncommutative space
}

\author{
Maja Burić Đand Voja Radovanovid \\ Faculty of Physics, P.O. Box 368, 11001 Belgrade, Yugoslavia
}

\begin{abstract}
In this paper we calculate the divergent part of the one loop effective action for QED on noncommutative space using the background field method. The effective action is obtained up to the second order in the noncommutative parameter $\theta$ and in the classical fields.
\end{abstract}

\footnotetext{
${ }^{1}$ E-mail: majab@ff.bg.ac.yu

${ }^{2}$ E-mail: rvoja@ff.bg.ac.yu
} 


\section{Introduction}

The discovery of noncommutative structures in string theory gave a considerable boost to the noncommutative field theories in the last few years, and put them into the focus of vigorous investigation. Besides the research on the various models appearing in the context of string theory, a lot of work has been devoted to the analysis of noncommutative (NC) theories for themselves. One approach is to formulate the theory in a representation-free manner, i. e. to build an abstract algebra of noncommuting coordinates with a defined set of relations, and then endow it with structures like derivations, forms, fields etc. [1, 2, 3]. The most commonly discussed is the canonical structure, defined by

$$
\left[\hat{x}^{\mu}, \hat{x}^{\nu}\right]=i \theta^{\mu \nu}
$$

$\hat{x}^{\mu}, \hat{x}^{\nu}$ are the elements of the algebra and $\theta^{\mu \nu}=-\theta^{\nu \mu}$ are constant complex numbers. The other structures like Lie algebra or quantum plane have been treated as well [1, 2]. The other possibility is to consider the representation of NC theory by the fields on commutative space, encoding the noncommutativity in the definition of multiplication. The multiplication which corresponds to the canonical structure (四) is the so-called Moyal-Weyl or $\star$-product:

$$
f \star g=\left.e^{\frac{i}{2} \theta^{\mu \nu} \frac{\partial}{\partial x^{\mu}} \frac{\partial}{\partial y^{\mu}}} f(x) g(y)\right|_{y \rightarrow x}
$$

where $f$ and $g$ are functions of the coordinates $x^{\mu}$. Obviously,

$$
\left[x^{\mu}, x^{\nu}\right]=x^{\mu} \star x^{\nu}-x^{\nu} \star x^{\mu}=i \theta^{\mu \nu} .
$$

The $\star$-product with its properties in integration provides a new class of actions characterized by dimensionfull parameter $\theta$, which have nonlocal lagrangians. In this setting the definition of noncommutative scalar field theories like $\Phi^{3}$ or $\Phi^{4}$ is straightforward. However, if one wants to define a gauge theory, the use of noncommutative multiplication rule imposes severe restrictions both on the choice of the gauge group and on the choice of its representation [4, 5, 6]. For example, in NC electrodynamics the values of charge are quantized and restricted to $\pm 1,0$.

The result of Seiberg and Witten [7] on the equivalence of certain commutative and noncommutative gauge theories shows that noncommutativity is not equivalent to quantization. Noncommutative field theories can be quantized in the conventional perturbative way [8, 9, 10] and their properties as unitarity and renormalizability can be analyzed (see 11, 12] for a more complete list of references). Several novel features appear, e.g. UV and IR sectors are mixed in the perturbative expansion. The UV-IR mixing can be seen from the fact that 'nonplanar' diagrams contain terms proportional to $|\tilde{p}|^{-n}$, with $\tilde{p}^{\mu}=\theta^{\mu \nu} p_{\nu}$.

Apparent nonrenormalizability of the NC theory might, in principle, be regained after the summation of the perturbation series. This was indeed shown for the NC 
$\Phi^{4}$ theory in 13 . However, it is a nontrivial task to prove a similar assertion for noncommutative $\mathrm{U}(1)$.

An obvious drawback of the perturbative treatment of $\mathrm{NC}$ theories is that the results, expressed as $|\tilde{p}|^{-n}$, are also nonperturbative in the parameter $\theta$. Therefore one cannot make a smooth commutative limit or estimate the effects of noncommutativity in the lowest order (in the sectors where they are small). In order to deal with this problem one may try to use the complementary approach of [1]. Namely, it was shown in [1] that, starting with the general abstract algebra, one can construct the representation of gauge symmetry for arbitrary group using the concept of 'covariant coordinates'. The gauge parameter and the gauge potential of the group are not Lie-algebra-valued any more, but take values in the enveloping algebra. If the generators of the gauge algebra are denoted by $T^{a}$, the following expansion of the gauge parameter $\hat{\Lambda}$ in powers of symmetrized products of $T^{a}$ holds:

$$
\hat{\Lambda}=\Lambda_{a} T^{a}+\Lambda_{a b}^{1}: T^{a} T^{b}:+\ldots+\Lambda_{a_{1} \ldots a_{n}}^{n-1}: T^{a_{1}} \ldots T^{a_{n}}:+\ldots .
$$

The gauge and matter fields are expanded in a similar way, [2]. The interesting point is that this expansion coincides with the expansion in $\theta$, if one maps the NC fields into the fields on commutative space via the Seiberg-Witten (SW) map [7]. The coefficients in the expansion depend on the derivatives of gauge and matter fields, so in some sense NC gauge theory is nonlocal both in spatial and gauge directions.

The SW map induces the $\theta$-expansion of the action as well. This gives the possibility to treat the $\theta$-linear term in the action as the first correction which describes the effects of noncommutativity in the lowest order. Further, $\theta$-expanded action enables one to approach the problem of quantization in a different way, i.e. considering lagrangian order by order in $\theta$. In this context, nonuniqueness of the SW map [14] takes the role of an additional 'symmetry' which might be used to prove renormalizability. This was done for noncommutative U(1) in [15]. The use of SW map also opens the possibility to circumvent the restrictions on the values of charges, e.g. in NC standard model [16].

Renormalizability of noncommutative QED with fermions seems to be a more complicated problem. It was discussed extensively in the papers 115, 17, 18 and one of their conclusions was that it would be of vital importance to find the exact form of all divergent $\theta$ and $\theta^{2}$ counterterms in the action. In the recent paper [19], Wulkenhaar found all $\theta$-linear divergencies in 2-, 3-, and 4-point functions diagramatically.

In this paper we calculate the divergent part of the one-loop effective action in the second order in the noncomutative parameter $\theta$ and in the classical fields, for noncommutative QED. We use the background field method. The plan of the paper is the following. In section 2 we briefly define our notation and fix the form of the lagrangian in the first order in $\theta$. In section 3 we give the basics of the background field method and outline how it will be applied to the given model. Section 4 presents the results for the effective action, while the appendix contains some helpful formulas. 


\section{Classical theory}

The noncommutative space which we use is $\mathbf{R}^{4}$ with the canonical structure

$$
\left[x^{\mu}, x^{\nu}\right]=i \theta^{\mu \nu}
$$

where $\mu, \nu=0, \ldots 3$ and $\star$ is the Moyal-Weyl product (2). The classical action for electrodynamics on this space is given by

$$
S=\int d^{4} x \hat{\bar{\psi}} \star\left(i \gamma^{\mu} \hat{D}_{\mu}-m\right) \hat{\psi}-\frac{1}{4} \int d^{4} x \hat{F}_{\mu \nu} \star \hat{F}^{\mu \nu}
$$

Here, $\hat{\psi}$ is the noncommutative fermionic matter field while $\hat{A}_{\mu}$ is the gauge potential. The corresponding field strength $\hat{F}_{\mu \nu}$ is defined as

$$
\hat{F}_{\mu \nu}=\partial_{\mu} \hat{A}_{\nu}-\partial_{\nu} \hat{A}_{\mu}-i\left(\hat{A}_{\mu} \star \hat{A}_{\nu}-\hat{A}_{\nu} \star \hat{A}_{\mu}\right)
$$

and the covariant derivative $\hat{D}_{\mu} \psi$ is

$$
\hat{D}_{\mu} \hat{\psi}=\partial_{\mu} \hat{\psi}-i \hat{A}_{\mu} \star \hat{\psi}
$$

It is clear that this theory is nonlocal.

The fields $\hat{\psi}, \hat{A}_{\mu}, \hat{F}_{\mu \nu}$ which give a representation of noncommutative electrodynamics can be, via the SW map, mapped into the representation of ordinary $\mathrm{U}(1)$. To the first order in $\theta$ the map is given by [2]:

$$
\begin{gathered}
\hat{A}_{\mu}=A_{\mu}-\frac{1}{2} \theta^{\rho \sigma} A_{\rho}\left(\partial_{\sigma} A_{\mu}+F_{\sigma \mu}\right), \\
\hat{\psi}=\psi-\frac{1}{2} \theta^{\mu \nu} A_{\mu} \partial_{\nu} \psi .
\end{gathered}
$$

Inserting (9) and (10) into the action (6), we get the classical $\theta$-expanded action [2, 15]

$$
S=S_{0}+S_{1, A}+S_{1, \psi}
$$

with

$$
\begin{gathered}
S_{0}=\int d^{4} x\left[\bar{\psi}\left(i \gamma^{\mu} D_{\mu}-m\right) \psi-\frac{1}{4} F^{\mu \nu} F_{\mu \nu}\right], \\
S_{1, A}=-\frac{1}{2} \theta^{\rho \sigma} \int d^{4} x\left[F_{\mu \rho} F_{\nu \sigma} F^{\mu \nu}-\frac{1}{4} F_{\rho \sigma} F_{\mu \nu} F^{\mu \nu}\right], \\
S_{1, \psi}=\frac{1}{2} \theta^{\rho \sigma} \int d^{4} x\left[-i F_{\mu \rho} \bar{\psi} \gamma^{\mu} D_{\sigma} \psi+\frac{1}{2} F_{\rho \sigma} \bar{\psi}\left(-i \gamma^{\mu} D_{\mu}+m\right) \psi\right] .
\end{gathered}
$$

The 'commutative' covariant derivative is $D_{\mu} \psi=\partial_{\mu} \psi-i A_{\mu} \psi$.

For the purpose of functional integration in the next section, we will express the Dirac spinor in terms of the Majorana spinors. They are introduced as $\psi_{1,2}=$ 
$\frac{1}{2}\left(\psi \pm \psi^{C}\right)$, where $\psi^{C}=C \bar{\psi}^{T}$ is the charge-conjugated spinor. The Dirac spinor is $\psi=\psi_{1}+i \psi_{2}$.

The action in terms of Majorana spinors reads:

$$
\begin{gathered}
S_{0}=\int d^{4} x\left[\bar{\psi}_{1}\left(i \gamma^{\mu} \partial_{\mu}-m\right) \psi_{1}+\bar{\psi}_{2}\left(i \gamma^{\mu} \partial_{\mu}-m\right) \psi_{2}\right. \\
\left.+i \bar{\psi}_{1} \gamma^{\mu} A_{\mu} \psi_{2}-i \bar{\psi}_{2} \gamma^{\mu} A_{\mu} \psi_{1}-\frac{1}{4} F^{\mu \nu} F_{\mu \nu}\right] \\
S_{1, \psi}=\frac{1}{2} \theta^{\rho \sigma} \int d^{4} x\left[\left(-\frac{i}{2} \bar{\psi}_{1} \gamma^{\mu}\left(F_{\mu \rho} \partial_{\sigma}+F_{\rho \sigma} \partial_{\mu}+F_{\sigma \mu} \partial_{\rho}\right) \psi_{1}+\frac{1}{2} m F_{\rho \sigma} \bar{\psi}_{1} \psi_{1}\right.\right. \\
-\frac{i}{2} \bar{\psi}_{2} \gamma^{\mu}\left(F_{\mu \rho} \partial_{\sigma}+F_{\rho \sigma} \partial_{\mu}+F_{\sigma \mu} \partial_{\rho}\right) \psi_{2}+\frac{1}{2} m F_{\rho \sigma} \bar{\psi}_{2} \psi_{2} \\
-\frac{i}{2} \bar{\psi}_{1} \gamma^{\mu}\left(F_{\mu \rho} A_{\sigma}+F_{\rho \sigma} A_{\mu}+F_{\sigma \mu} A_{\rho}\right) \psi_{2} \\
\left.+\frac{i}{2} \bar{\psi}_{2} \gamma^{\mu}\left(F_{\mu \rho} A_{\sigma}+F_{\rho \sigma} A_{\mu}+F_{\sigma \mu} A_{\rho}\right) \psi_{1}\right]
\end{gathered}
$$

The cyclic combinations which appear in (17) will be in the following written in a compact way

$$
\begin{gathered}
F_{\mu \rho} A_{\sigma}+F_{\rho \sigma} A_{\mu}+F_{\sigma \mu} A_{\rho}=\frac{1}{2} \Delta_{\sigma \rho \mu}^{\alpha \beta \gamma} F_{\gamma \beta} A_{\alpha}, \\
F_{\mu \rho} \partial_{\sigma}+F_{\rho \sigma} \partial_{\mu}+F_{\sigma \mu} \partial_{\rho}=\frac{1}{2} \Delta_{\sigma \rho \mu}^{\alpha \beta \gamma} F_{\gamma \beta} \partial_{\alpha},
\end{gathered}
$$

introducing the symbol $\Delta_{\sigma \rho \mu}^{\alpha \beta \gamma}$. $\Delta_{\sigma \rho \mu}^{\alpha \beta \gamma}$ is cyclic separately in upper and lower indices, and antisymmetric in any pair of upper or lower indices:

$$
\Delta_{\sigma \rho \mu}^{\alpha \beta \gamma}=\delta_{\sigma}^{\alpha} \delta_{\rho}^{\beta} \delta_{\mu}^{\gamma}-\delta_{\rho}^{\alpha} \delta_{\sigma}^{\beta} \delta_{\mu}^{\gamma}+(\operatorname{cyclic} \alpha \beta \gamma)=-\epsilon^{\alpha \beta \gamma \lambda} \epsilon_{\sigma \rho \mu \lambda}
$$

\section{Background field method}

In order to find the divergent part of the one-loop effective action we will use the background field method. Let us introduce it briefly [20, 21, 22]. If we consider a theory described by a set of fields $\phi^{i}$ with the classical action $S\left[\phi^{i}\right]$ and sources $J_{i}$, the generating functional $W\left[J_{i}\right]$ for the connected Green functions is given by

$$
e^{i W\left[J_{i}\right]}=\int \prod \mathcal{D} \phi_{i} e^{i\left(S\left[\phi^{i}\right]+\int d x J_{i} \phi^{i}\right)}
$$

\footnotetext{
${ }^{3}$ There is a number of identities which Majorana spinors satisfy and which we use. To encounter some of them: let $\phi, \chi$ be Majorana spinors. Then: $\bar{\phi} \chi=\bar{\chi} \phi ; \bar{\phi} \gamma_{\mu} \chi=-\bar{\chi} \gamma_{\mu} \phi$; $\bar{\phi} \sigma_{\mu \nu} \chi=-\bar{\chi} \sigma_{\mu \nu} \phi ; \bar{\phi} \gamma_{5} \chi=\bar{\chi} \gamma_{5} \phi ; \bar{\phi} \gamma_{\mu} \gamma_{5} \chi=\bar{\chi} \gamma_{\mu} \gamma_{5} \phi$.
} 
The field components $\phi_{i}$ can be fermionic or/and bosonic. The effective action,

$$
\Gamma\left[\phi_{0}^{i}\right]=W\left[J_{i}\right]-\int d x J_{i} \phi_{0}^{i},
$$

is a Legendre transformation of the generating functional $W\left[J_{i}\right] . \phi_{0}^{i}$ are the solutions of the classical equations of motion:

$$
J_{i}=-\frac{\delta_{R} \Gamma}{\delta \phi_{0}^{i}}=-\frac{\delta_{R} S}{\delta \phi_{0}^{i}},
$$

where $\delta_{R} / \delta$ is the right functional derivative. From (18) and (19) we get

$$
e^{i \Gamma\left[\phi_{0}^{i}\right]}=\int \prod \mathcal{D} \phi_{i} e^{i\left(S\left[\phi^{i}\right]+\int d x J_{i}\left(\phi^{i}-\phi_{0}^{i}\right)\right)} .
$$

In order to evaluate this integral we decompose the field $\phi^{i}$ as

$$
\phi^{i}=\phi_{0}^{i}+\Phi^{i},
$$

where $\Phi_{i}$ are the quantum fluctuations around the classical configuration. The functional integral (21) is then calculated by the saddle-point method after the Taylor expansion:

$$
e^{i \Gamma\left[\phi_{0}^{i}\right]} \approx e^{i S\left[\phi_{0}^{i}\right]} \int \prod \mathcal{D} \Phi_{i} e^{\frac{i}{2} \int \Phi^{i} S_{i j}^{(2)}\left(\phi_{0}\right) \Phi^{j}},
$$

where $S_{i j}^{(2)}=\left.\frac{\delta_{L}}{\delta \phi^{i}} \frac{\delta_{R}}{\delta \phi^{j}} S\right|_{\phi^{i}=\phi_{0}^{i}}$. The result is the one-loop effective action

$$
\Gamma\left[\phi_{0}^{i}\right]=S\left[\phi_{0}^{i}\right]-\frac{1}{2 i} \mathrm{~S} \operatorname{Tr}\left(\log S^{(2)}\left[\phi_{0}^{i}\right]\right),
$$

$\mathrm{STr}$ is the functional supertrace.

Let us discuss how to apply the background field method in the case of $\mathrm{NC}$ electrodynamics. The fields in the theory are the real vector $A_{\mu}$ and the Dirac spinor $\psi$ and they are coupled. In order to perform the functional integration we have to put them into one 'multiplet' field. However, $A_{\mu}$ are real-number valued while $\psi$ are complex-Grassmann valued (if they were independent they would have entered the effective action with different coefficients $-\frac{1}{2}$ and 1 ). To make all fields 'real' we need to express Dirac spinor $\psi$ in terms of two Majorana spinors $\psi_{1}$ and $\psi_{2}$.

Denoting now the quantum corrections now by $\mathcal{A}^{\mu}, \Psi$ and splitting

$$
A^{\mu} \rightarrow A^{\mu}+\mathcal{A}^{\mu} \quad, \psi \rightarrow \psi+\Psi
$$

we obtain for the quadratic part of the action the expression of the type

$$
S^{(2)}=\int d^{4} x\left(\begin{array}{lll}
\mathcal{A}_{\alpha} & \bar{\Psi}_{1} & \bar{\Psi}_{2}
\end{array}\right) \mathcal{B}\left(\begin{array}{c}
\mathcal{A}_{\beta} \\
\Psi_{1} \\
\Psi_{2}
\end{array}\right),
$$


where the matrix $\mathcal{B}$ contains classical fields. We have to include in $\mathcal{B}$ the gauge fixing term,

$$
S_{\mathrm{GF}}=-\frac{1}{2} \int d^{4} x\left(\partial_{\mu} \mathcal{A}^{\mu}\right)^{2},
$$

while the ghost action will not contribute. The one-loop effective action is then

$$
\Gamma_{1}=\frac{i}{2} \log \operatorname{Sdet} \mathcal{B}=\frac{i}{2} \mathrm{~S} \operatorname{Tr} \log \mathcal{B} .
$$

Let us consider some properties of the matrix $\mathcal{B}$. It can be written in a $3 \times 3$ block-matrix form

$$
\mathcal{B}=\left(\begin{array}{lll}
\mathcal{B}_{11} & \mathcal{B}_{12} & \mathcal{B}_{13} \\
\mathcal{B}_{21} & \mathcal{B}_{22} & \mathcal{B}_{23} \\
\mathcal{B}_{31} & \mathcal{B}_{32} & \mathcal{B}_{33}
\end{array}\right),
$$

where the submatrices $\mathcal{B}_{12}, \mathcal{B}_{13}, \mathcal{B}_{21}$ and $\mathcal{B}_{31}$ are Grassmann-odd while the rest are Grassmann-even. The supertrace of $\mathcal{B}$ is defined by

$$
\mathrm{S} \operatorname{Tr} \mathcal{B}=\operatorname{Tr} \mathcal{B}_{11}-\operatorname{Tr} \mathcal{B}_{22}-\operatorname{Tr} \mathcal{B}_{33}
$$

$\mathcal{B}$ is of the form

$$
\mathcal{B}=\left(\begin{array}{ccc}
\frac{1}{2} g_{\alpha \beta} \square & 0 & 0 \\
0 & i \not & 0 \\
0 & 0 & i \not \phi
\end{array}\right)+\mathcal{M} .
$$

In order to expand $\log \mathcal{B}$ around identity, we multiply it by the matrix $\mathcal{C C}^{-1}$ [22], with

$$
\mathcal{C}=\left(\begin{array}{ccc}
2 & 0 & 0 \\
0 & -i \not \partial & 0 \\
0 & 0 & -i \not \partial
\end{array}\right) .
$$

Then

$$
\begin{aligned}
\Gamma_{1} & =\frac{i}{2} \mathrm{~S} \operatorname{Tr} \log (\mathcal{B C})+\frac{i}{2} \mathrm{~S} \operatorname{Tr} \log \mathcal{C}^{-1} \\
& =\frac{i}{2} \mathrm{~S} \operatorname{Tr}\left(\mathcal{I}+\square^{-1} \mathcal{M C}\right)+\frac{i}{2} \mathrm{~S} \operatorname{Tr} \log \mathcal{C}^{-1}+\frac{i}{2} \mathrm{~S} \operatorname{Tr} \log \square
\end{aligned}
$$

where $\mathcal{I}=\operatorname{diag}\left(g_{\mu \nu}, 1,1\right)$. As usual, the second and the third terms, being independent on the fields, can be included in infinite renormalization. Note that the propagator for all fields is now $\square^{-1}$, while the massive fermionic terms are in the interaction part, $\mathcal{M}$.

Performing the transformations described above, for NC QED we obtain the effective action in the following form:

$$
\Gamma=S_{0}+\frac{i}{2} \operatorname{STr} \log \left(\mathcal{I}+\square^{-1} N_{0}+\square^{-1} N_{1}+\square^{-1} T_{1}+\square^{-1} T_{2}\right) .
$$


The matrices $N_{0}, N_{1}, T_{1}$ and $T_{2}$ are given by

$$
\begin{aligned}
& N_{0}=\left(\begin{array}{ccc}
0 & 0 & 0 \\
0 & i m \not & 0 \\
0 & 0 & i m \not
\end{array}\right) \\
& N_{1}=\left(\begin{array}{ccc}
0 & -i \bar{\psi} \gamma^{\alpha} \not \partial & \bar{\psi} \gamma^{\alpha} \not \partial \\
2 \gamma^{\beta} \psi & 0 & \not A \not \partial \\
-2 i \gamma^{\beta} \psi & -\not \not \partial & 0
\end{array}\right) \\
& T_{1}=\left(\begin{array}{ccc}
V & A_{1} & A_{2} \\
B_{1} & C & 0 \\
B_{2} & 0 & C
\end{array}\right) \\
& T_{2}=\theta^{\rho \sigma} \Delta_{\sigma \rho \mu}^{\alpha \beta \gamma}\left(\begin{array}{ccc}
-\frac{1}{2} \bar{\psi} \gamma^{\mu} \psi \partial_{\gamma} & \frac{i}{4}\left(\frac{1}{2} F_{\gamma \beta}+\partial_{\gamma} A_{\beta}\right) \bar{\psi} \gamma^{\mu} \not \partial & -\frac{1}{4}\left(\frac{1}{2} F_{\gamma \beta}+\partial_{\gamma} A_{\beta}\right) \bar{\psi} \gamma^{\mu} \not \partial \\
-\frac{1}{2} \gamma^{\mu} \psi\left(-\frac{1}{2} F_{\gamma \alpha}+A_{\alpha} \partial_{\gamma}\right) & \frac{i}{8} A_{\alpha} F_{\gamma \beta} \gamma^{\mu} \not \partial & -\frac{1}{8} A_{\alpha} F_{\gamma \beta} \gamma^{\mu} \not \supset \\
\frac{i}{2} \gamma^{\mu} \psi\left(-\frac{1}{2} F_{\gamma \alpha}+A_{\alpha} \partial_{\gamma}\right) & \frac{1}{8} A_{\alpha} F_{\gamma \beta} \gamma^{\mu} \not \partial & \frac{i}{8} A_{\alpha} F_{\gamma \beta} \gamma^{\mu} \not \partial
\end{array}\right)
\end{aligned}
$$

where $A_{i}, B_{i}(i=1,2)$ and $C$ are

$$
\begin{aligned}
A_{i} & =\frac{i}{4} \theta^{\rho \sigma} \Delta_{\sigma \rho \mu}^{\alpha \beta \gamma} \partial_{\gamma}\left(-\left(\partial_{\beta} \bar{\psi}_{i}\right) i \gamma^{\mu}-\frac{m}{2} \delta_{\beta}^{\mu} \bar{\psi}_{i}\right) \not \partial \\
B_{i} & =\frac{1}{2} \theta^{\rho \sigma} \Delta_{\sigma \rho \mu}^{\alpha \beta \gamma}\left(-i \gamma^{\mu}\left(\partial_{\alpha} \psi_{i}\right)+\frac{m}{2} \delta_{\alpha}^{\mu} \psi_{i}\right) \partial_{\gamma} \\
C & =-\frac{i}{4} \theta^{\rho \sigma}\left(-\frac{i}{2} \Delta_{\sigma \rho \mu}^{\alpha \beta \gamma} \gamma^{\mu} F_{\gamma \beta} \partial_{\alpha}+m F_{\rho \sigma}\right) \not \partial .
\end{aligned}
$$

$$
\begin{aligned}
& V=\overleftarrow{\partial_{\mu}} V^{\mu \alpha, \nu \beta}(x) \overrightarrow{\partial_{\beta}} \text { comes from the term }\left(\partial_{\mu} \mathcal{A}_{\alpha}\right) V^{\mu \alpha, \nu \beta}\left(\partial_{\nu} \mathcal{A}_{\beta}\right) \text { in } S^{(2)}: \\
& \qquad \begin{aligned}
V^{\mu \rho, \nu \sigma} & =\frac{1}{2}\left(g^{\mu \nu} g^{\rho \sigma}-g^{\mu \sigma} g^{\nu \rho}\right) \theta^{\alpha \beta} F_{\alpha \beta} \\
& +g^{\mu \nu}\left(\theta^{\alpha \rho} F^{\sigma}{ }_{\alpha}+\theta^{\alpha \sigma} F^{\rho}{ }_{\alpha}\right)+g^{\rho \sigma}\left(\theta^{\alpha \mu} F^{\nu}{ }_{\alpha}+\theta^{\alpha \nu} F^{\mu}{ }_{\alpha}\right) \\
& -g^{\mu \sigma}\left(\theta^{\alpha \rho} F^{\nu}{ }_{\alpha}+\theta^{\alpha \nu} F^{\rho}{ }_{\alpha}\right)-g^{\nu \rho}\left(\theta^{\alpha \sigma} F^{\mu}{ }_{\alpha}+\theta^{\alpha \mu} F^{\sigma}{ }_{\alpha}\right) \\
& +\theta^{\mu \rho} F^{\nu \sigma}+\theta^{\nu \sigma} F^{\mu \rho}-\theta^{\rho \sigma} F^{\mu \nu}-\theta^{\mu \nu} F^{\rho \sigma}-\theta^{\nu \rho} F^{\mu \sigma}-\theta^{\mu \sigma} F^{\nu \rho}
\end{aligned}
\end{aligned}
$$

We will also use $V_{\rho \sigma}^{\mu \nu}=g_{\rho \alpha} g_{\sigma \beta} V^{\mu \alpha, \nu \beta}$ which has the obvious symmetry $V_{\mu \nu}^{\alpha \beta}=V_{\nu \mu}^{\beta \alpha}$.

Note that in $N_{1}$ and $T_{2}$ the Majorana spinors $\psi_{1}$ and $\psi_{2}$ add up neatly to the Dirac spinor $\psi$. $T_{1}$ depends on both of the Majorana spinors explicitly, but in the same way. These properties ensure that the final results will be expressed in terms of the Dirac spinor.

\section{Divergent one-loop effective action}

The operator $\mathcal{B C}$ in the formula (29) is splitted in a way convenient for the analysis of perturbation series. Let us explain the notation a little further. $T$-matrices are linear 
in the parameter $\theta$. Index denotes the number of classical fields in a given matrix, i.e. in diagrammatic language, shows the number of 'external legs' of the corresponding diagram. In our calculation we confine to the corrections of linear and quadratic order in $\theta$ and of the second order in classical fields. If we consider the expansion of (29)

$$
\begin{aligned}
\Gamma_{1} & =\frac{i}{2} \mathrm{~S} \operatorname{Tr} \log \left(1+\square^{-1} N_{0}+\square^{-1} N_{1}+\square^{-1} T_{1}+\square^{-1} T_{2}\right) \\
& =\frac{i}{2} \sum_{n=1}^{\infty} \frac{(-1)^{n+1}}{n} \mathrm{~S} \operatorname{Tr}\left(\square^{-1} N_{0}+\square^{-1} N_{1}+\square^{-1} T_{1}+\square^{-1} T_{2}\right)^{n},
\end{aligned}
$$

it seems as we have to include only $n=1,2$. But due to the nonvanishing fermionic mass $m$ (i.e. the existence of the term $N_{0}$ ), in principle we will have to take into account also higher powers of $n$. $n$ will be determined from the fact that we are calculating only the divergent part. Analyzing the structure of $\square^{-1} N_{0}, \ldots, \square^{-1} T_{2}$ in some detail we conclude that the following terms in the expansion (33) may be divergent: $\left(\square^{-1} N_{0}\right)^{k} \square^{-1} T_{2}$ for $k=2,3,4 ;\left(\square^{-1} N_{0}\right)^{k} \square^{-1} N_{1} \square^{-1} T_{1}$ for $k=1,2,3$; $\left(\square^{-1} N_{0}\right)^{k}\left(\square^{-1} N_{1}\right)^{2}$ for $k=1,2$ and $\left(\square^{-1} N_{0}\right)^{k}\left(\square^{-1} T_{1}\right)^{2}$ for $k=1,2,3,4$. (Here, of course, terms are written symbolically i. e. without the exact order of the operators.) It is also clear that in the massless fermionic case the absence of $N_{0}$ brings a considerable simplification.

Let us first discuss maximally simplified situation: the purely bosonic case. Then $N_{0}, N_{1}$ and $T_{2}$ are all absent, while $T_{1}$ reduces to $\mathcal{T}_{1}$ :

$$
\mathcal{T}_{1}=\left(\begin{array}{lll}
V & 0 & 0 \\
0 & 0 & 0 \\
0 & 0 & 0
\end{array}\right)
$$

We have then

$$
\Gamma_{b}=\frac{i}{2} \mathrm{~S} \operatorname{Tr} \log \left(1+\square^{-1} \mathcal{T}_{1}\right)=\frac{i}{2}\left[\operatorname{Tr} \square^{-1} \mathcal{T}_{1}-\frac{1}{2} \operatorname{Tr}\left(\square^{-1} \mathcal{T}_{1}\right)^{2}+\ldots\right]
$$

and in the $\theta^{2}, A^{2}$-order we need only first two terms. Even this calculation proves to be technically difficult, mainly due to the complicated tensorial structure of $V$.

It can be easily seen that the divergent part of the first trace in (35), $\operatorname{Tr} \square^{-1} \mathcal{T}_{1}$ vanishes. The second term in (35) is

$$
\int d^{4} x d^{4} y g^{\nu \rho} g^{\mu \sigma} V_{\nu \mu}^{\alpha \beta}(x) V_{\sigma \rho}^{\gamma \delta}(y) \partial_{\alpha}^{x} \partial_{\delta}^{y} G(y-x) \partial_{\beta}^{x} \partial_{\gamma}^{y} G(x-y),
$$

where

$$
G(x-y)=-\int \frac{d^{4} k}{(2 \pi)^{4}} \frac{e^{-i k(x-y)}}{k^{2}}
$$

is the Green function which satisfies

$$
\square_{x} G(x-y)=\delta^{(4)}(x-y)
$$


After Fourier transformation, the expression (36) becomes

$$
\frac{1}{(2 \pi)^{8}} \int d^{4} p d^{4} k g^{\nu \rho} g^{\mu \sigma} V_{\nu \mu}^{\alpha \beta}(p) V_{\sigma \rho}^{\gamma \delta}(-p) \frac{k_{\beta} k_{\gamma}(k+p)_{\alpha}(k+p)_{\delta}}{k^{2}(k+p)^{2}} .
$$

The dimensional regularization in $D=4-\epsilon$-dimensional space gives for the divergent part of (36)

$$
\begin{aligned}
& \frac{i \pi^{\frac{D}{2}}}{2 \epsilon} \int \frac{d^{4} p}{(2 \pi)^{8}} g^{\nu \rho} g^{\mu \sigma} V_{\nu \mu}^{\alpha \beta}(p) V_{\sigma \rho}^{\gamma \delta}(-p)\left[\frac{2}{15} p_{\alpha} p_{\beta} p_{\gamma} p_{\delta}\right. \\
+ & \frac{1}{15} p^{2}\left(g_{\alpha \beta} p_{\gamma} p_{\delta}+g_{\alpha \gamma} p_{\beta} p_{\delta}+g_{\alpha \delta} p_{\beta} p_{\gamma}+g_{\gamma \beta} p_{\alpha} p_{\delta}+g_{\delta \beta} p_{\alpha} p_{\gamma}+g_{\gamma \delta} p_{\alpha} p_{\delta}\right) \\
+ & \frac{1}{60}\left(g_{\alpha \beta} g_{\gamma \delta}+g_{\alpha \delta} g_{\beta \gamma}+g_{\alpha \gamma} g_{\beta \delta}\right) p^{4} \\
- & \left.\frac{1}{6}\left(g_{\beta \gamma} p_{\alpha} p_{\delta}-g_{\alpha \delta} p_{\beta} p_{\gamma}\right) p^{2}\right] .
\end{aligned}
$$

Rewriting (40) in the coordinate space we obtain the one-loop correction:

$$
\begin{aligned}
\Gamma_{b} & =\frac{1}{128 \pi^{2} \epsilon} g^{\nu \rho} g^{\mu \sigma} \int d^{4} x\left[\frac{2}{15} \partial_{\alpha} \partial_{\beta} V_{\nu \mu}^{\alpha \beta} \partial_{\gamma} \partial_{\delta} V_{\sigma \rho}^{\gamma \delta}-\frac{1}{3} g_{\beta \gamma} \square V_{\nu \mu}^{\alpha \beta} \partial_{\alpha} \partial_{\delta} V_{\sigma \rho}^{\gamma \delta}\right. \\
& +\frac{2}{15}\left(g_{\alpha \beta} \square V_{\nu \mu}^{\alpha \beta} \partial_{\gamma} \partial_{\delta} V_{\sigma \rho}^{\gamma \delta}+g_{\alpha \gamma} \square V_{\nu \mu}^{\alpha \beta} \partial_{\beta} \partial_{\delta} V_{\sigma \rho}^{\gamma \delta}+g_{\alpha \delta} \square V_{\nu \mu}^{\alpha \beta} \partial_{\gamma} \partial_{\delta} V_{\sigma \rho}^{\gamma \delta}\right) \\
& \left.+\frac{1}{60}\left(g_{\alpha \beta} g_{\gamma \delta}+g_{\alpha \delta} g_{\beta \gamma}+g_{\alpha \gamma} g_{\beta \delta}\right) \square V_{\nu \mu}^{\alpha \beta} \square V_{\rho \sigma}^{\gamma \delta}\right] .
\end{aligned}
$$

The hard part now is to introduce the explicit form of matrix elements (33) and perform the index summations. The result reads

$$
\begin{aligned}
\Gamma_{b} & =\frac{1}{64 \pi^{2} \epsilon} \int d^{4} x\left[\frac{12}{5} \square \tilde{F}_{\mu \nu} \square \tilde{F}^{\mu \nu}+\frac{2}{5} \square \tilde{F}_{\mu \nu} \square \tilde{F}^{\nu \mu}-\frac{1}{60}(\square \tilde{F})^{2}\right. \\
& +\frac{7}{15} \square \tilde{F} \partial_{\mu} \partial_{\nu} \tilde{F}^{\mu \nu}+\frac{3}{5} \square \tilde{F}_{\nu \mu} \partial^{\nu} \partial_{\sigma} \tilde{F}^{\sigma \mu}-\frac{4}{5} \square \tilde{F}_{\nu \mu} \partial^{\nu} \partial_{\sigma} \tilde{F}^{\mu \sigma} \\
& -\frac{7}{5} \square \tilde{F}_{\nu \mu} \partial^{\mu} \partial_{\sigma} \tilde{F}^{\nu \sigma}+\frac{8}{15}\left(\partial_{\mu} \partial_{\nu} \tilde{F}^{\mu \nu}\right)^{2} \\
& \left.-\frac{1}{2} \theta^{2} \square F^{\alpha \nu} \partial_{\alpha} \partial_{\delta} F_{\nu}^{\delta}-\frac{1}{2} \square F_{\nu \mu} \tilde{\partial}^{\alpha} \tilde{\partial}_{\alpha} F^{\nu \mu}\right],
\end{aligned}
$$

where we use the notation

$$
\tilde{F}^{\mu \nu}=\theta_{\alpha}^{\mu} F^{\alpha \nu}, \quad \tilde{F}=\theta_{\mu \nu} F^{\mu \nu}, \quad \tilde{\partial}^{\mu}=\theta_{\alpha}^{\mu} \partial^{\alpha}, \quad \theta^{2}=\theta^{\rho \sigma} \theta_{\rho \sigma} .
$$

In order to simplify further we need the Bianchi identities; they are given in the convenient form in the appendix. Finally, the divergent part of the U(1) effective action becomes

$$
\begin{aligned}
\Gamma_{b} & =\frac{1}{64 \pi^{2} \epsilon} \int d^{4} x\left[\square \tilde{F}^{\mu \nu} \square \tilde{F}_{\mu \nu}+\frac{2}{15} \square \tilde{F}^{\mu \rho} \partial_{\mu} \partial^{\nu} \tilde{F}_{\nu \rho}\right. \\
& \left.+\frac{1}{5} \square F^{\mu \nu} \tilde{\partial}_{a} \tilde{\partial}^{\alpha} F_{\mu \nu}-\frac{1}{4} \theta^{2} \square F^{\mu \nu} \square F_{\mu \nu}\right] .
\end{aligned}
$$


This result coincides exactly with the result of [15]. To conclude: working out the bosonic part separately we have not only done a lengthy piece of calculation, but we have also performed an important check.

Let us pass now to the full QED case. In the lowest nonvanishing order, corresponding to $n=2$ in the expression (33), for the correction containing two classical fields we get

$$
\begin{aligned}
\Gamma_{1}^{\prime} & =-\frac{i}{4} \mathrm{~S} \operatorname{Tr}\left(\square^{-1} N_{0}+\square^{-1} N_{1}+\square^{-1} T_{1}+\square^{-1} T_{2}\right)^{2} \\
& =-\frac{i}{4} \mathrm{~S} \operatorname{Tr}\left(\left(\square^{-1} N_{0}+\square^{-1} N_{1}\right)^{2}+2\left(\square^{-1} N_{0}+\square^{-1} N_{1}\right) \square^{-1} T_{1}+\left(\square^{-1} T_{1}\right)^{2}\right)
\end{aligned}
$$

The relevant supertraces (i.e., their $\frac{1}{\epsilon}$-parts) are:

$$
\begin{aligned}
& \operatorname{STr}\left(\square^{-1} N_{0}+\square^{-1} N_{1}\right)^{2}=\frac{i}{(4 \pi)^{2} \epsilon}\left(-\frac{8}{3} F_{\mu \nu} F^{\mu \nu}+16 i \bar{\psi} \not \partial\right), \\
& 2 \mathrm{~S} \operatorname{Tr}\left(\square^{-1} N_{0}+\square^{-1} N_{1}\right) \square^{-1} T_{1}=\frac{i}{(4 \pi)^{2} \epsilon} \theta^{\rho \sigma}\left[\frac{4}{3} \bar{\psi} \gamma_{\rho} \partial_{\sigma}(\square-i m \not \partial)+\frac{2}{3} \bar{\psi} \sigma_{\rho \sigma} \square(i \not \partial-m) \psi\right], \\
& \mathrm{STr}\left(\square^{-1} T_{1}\right)^{2}=\frac{i}{(4 \pi)^{2} \epsilon}\left[\frac { i } { 1 2 } \left(2 \theta_{\mu}^{\rho} \theta^{\mu \sigma} \bar{\psi} \partial_{\rho} \partial_{\sigma} \not \partial \square \psi+4 \theta^{\rho}{ }_{\mu} \theta^{\mu \sigma} \bar{\psi} \gamma_{\rho} \partial_{\sigma} \square^{2} \psi+\theta^{2} \bar{\psi} \not \square^{2} \psi\right.\right. \\
&+2 i m \theta^{2} \bar{\psi} \square^{2} \psi \\
&\left.-2 m^{2} \theta^{\rho}{ }_{\mu} \theta^{\mu \sigma} \bar{\psi} \partial_{\rho} \partial_{\sigma} \not \partial \psi-2 m^{2} \theta^{\rho}{ }_{\mu} \theta^{\mu \sigma} \bar{\psi} \gamma_{\rho} \partial_{\sigma} \square \psi-m^{2} \theta^{2} \bar{\psi} \not \supset \square\right) \\
&\left.-\frac{m^{2}}{2} \tilde{F} \square \tilde{F}-\frac{1}{30} \tilde{F}^{\rho \sigma} \square^{2} \tilde{F}_{\sigma \rho}+\frac{2}{15} \tilde{F}^{\rho \sigma} \square^{2} \tilde{F}_{\rho \sigma}-\frac{2}{15} \tilde{F}^{\rho \sigma} \square \partial_{\mu} \partial^{\nu} \tilde{F}_{\rho \nu}\right] \\
&+V V \operatorname{term} .
\end{aligned}
$$

$V V$ is the bosonic correction previously found. $\Gamma_{1}^{\prime}$ is now easily obtained summing up 4648 .

The list of supertraces necessary to find the higher contributions, $n=3, \ldots$, is:

$$
\begin{aligned}
& \mathrm{S} \operatorname{Tr}\left(\square^{-1} N_{0}\right)^{k} \square^{-1} T_{2}=0 \text { for } k=1,2,3,4 \text {, } \\
& \mathrm{S} \operatorname{Tr} \square^{-1} N_{0}\left(\square^{-1} N_{1}\right)^{2}=\frac{i}{(4 \pi)^{2} \epsilon} 32 m \int d^{4} x \bar{\psi} \psi \\
& 4 \mathrm{~S} \operatorname{Tr}\left(\square^{-1} N_{0}\right)^{2}\left(\square^{-1} N_{1}\right)^{2}+2 \mathrm{~S} \operatorname{Tr} \square^{-1} N_{0} \square^{-1} N_{1} \square^{-1} N_{0} \square^{-1} N_{1}=0, \\
& \operatorname{STr}\left[\left(\square^{-1} N_{0}\right)^{2} \square^{-1} N_{1} \square^{-1} T_{1}+\left(\square^{-1} N_{0}\right)^{2} \square^{-1} T_{1} \square^{-1} N_{1}+\square^{-1} N_{0} \square^{-1} N_{1} \square^{-1} N_{0} \square^{-1} T_{1}\right] \\
& =\frac{i}{(4 \pi)^{2} \epsilon} \theta^{\rho \sigma} \int d^{4} x\left(2 m^{2} \bar{\psi} \gamma_{\rho} \partial_{\sigma} \psi+i m^{2} \bar{\psi} \gamma_{\rho} \gamma_{\sigma}(i \not \partial-m) \psi\right)
\end{aligned}
$$




$$
\begin{aligned}
& \mathrm{S} \operatorname{Tr} \square^{-1} N_{0}\left(\square^{-1} T_{1}\right)^{2}=\frac{i}{(4 \pi)^{2} \epsilon} \int d^{4} x\left(\frac{m^{3}}{3} \theta^{\rho}{ }_{\mu} \theta^{\mu \sigma} \bar{\psi} \partial_{\rho} \partial_{\sigma} \psi+\frac{m}{6} \theta^{\rho}{ }_{\mu} \theta^{\mu \sigma} \bar{\psi} \partial_{\rho} \partial_{\sigma} \square \psi(53)\right. \\
& -\frac{i m^{2}}{3} \theta^{\rho}{ }_{\mu} \theta^{\mu \sigma} \bar{\psi} \gamma_{\rho} \partial_{\sigma} \square \psi+\frac{m^{3}}{12} \theta^{2} \bar{\psi} \square \psi-\frac{i m^{2}}{6} \theta^{2} \bar{\psi} \square \not \partial \psi \\
& \left.-\frac{m}{12} \theta^{2} \bar{\psi} \square^{2} \psi-\frac{m^{2}}{3} \tilde{F} \square \tilde{F}\right) \text {, } \\
& \operatorname{STr}\left(\square^{-1} N_{0}\right)^{2}\left(\square^{-1} T_{1}\right)^{2}=\frac{i}{(4 \pi)^{2} \epsilon} m^{2} \int d^{4} x\left(\frac{m}{3} \theta_{\mu}^{\rho} \theta^{\mu \sigma} \bar{\psi} \partial_{\rho} \partial_{\sigma} \psi-\frac{m}{6} \theta^{2} \bar{\psi} \square \psi\right. \\
& -\frac{i m^{2}}{3} \theta_{\mu}^{\rho} \theta^{\mu \sigma} \bar{\psi} \gamma_{\sigma} \partial_{\rho} \psi+\frac{i}{6} \theta_{\mu}^{\rho} \theta^{\mu \sigma} \bar{\psi} \partial_{\rho} \partial_{\sigma} \not \partial \psi \\
& +\frac{i}{3} \theta_{\mu}^{\rho} \theta^{\mu \sigma} \bar{\psi} \gamma_{\rho} \partial_{\sigma} \square \psi+\frac{i}{12} \theta^{2} \bar{\psi} \not \partial \square \psi-\frac{i m^{2}}{12} \theta^{2} \bar{\psi} \not \partial \psi \\
& \text { - } m^{2} \tilde{F}^{2}-\frac{1}{12} \tilde{F} \square \tilde{F}+\frac{1}{2} \tilde{F}^{\rho \sigma} \square \tilde{F}_{\rho \sigma} \\
& \left.-\frac{1}{3} \tilde{F}^{\rho \sigma} \partial_{\sigma} \partial^{\nu} \tilde{F}_{\rho \nu}-\frac{1}{6} \tilde{F}^{\rho \sigma} \square \tilde{F}_{\sigma \rho}\right), \\
& \operatorname{STr}\left(\square^{-1} N_{0}\right)^{4}\left(\square^{-1} T_{1}\right)^{2}=\operatorname{STr}\left(\left(\square^{-1} N_{0}\right)^{2} \square^{-1} T_{1}\left(\square^{-1} N_{0}\right)^{2} \square^{-1} T_{1}\right) \\
& =-\frac{i}{(4 \pi)^{2} \epsilon} m^{4} \int d^{4} x\left(-\frac{2}{3} \tilde{F}_{\mu \nu} \tilde{F}^{\mu \nu}+\frac{1}{3} \tilde{F}^{2}+\frac{1}{3} \tilde{F}_{\mu \nu} \tilde{F}^{\nu \mu}\right) \text {, } \\
& \operatorname{STr}\left(\square^{-1} N_{0}\right)^{3}\left(\square^{-1} T_{1}\right) \square^{-1} N_{0} \square^{-1} T_{1}=-\frac{i}{(4 \pi)^{2} \epsilon} m^{4} \int d^{4} x \tilde{F}_{\mu \nu} \tilde{F}^{\mu \nu}
\end{aligned}
$$

Expanding the logarithm under the supertrace and using the previous results for the higher-order contribution we obtain:

$$
\begin{aligned}
\Gamma_{1}^{\prime \prime} & =\frac{i}{(4 \pi)^{2} \epsilon} \frac{1}{2} \int d^{4} x\left[32 m i \bar{\psi} \psi-i m^{2} \theta^{\rho \sigma}\left(2 \bar{\psi} \gamma_{\rho} \partial_{\sigma} \psi+i \bar{\psi} \gamma_{\rho} \gamma_{\sigma}(i \not \partial-m) \psi\right)\right. \\
& +\frac{i m^{3}}{2} \theta^{\rho}{ }_{\mu} \theta^{\mu \sigma} \bar{\psi} \partial_{\rho} \partial_{\sigma} \psi+\frac{2 m^{2}}{3} \theta_{\mu}^{\rho} \theta^{\mu \sigma} \bar{\psi} \gamma_{\rho} \partial_{s} \square \psi \\
& +\frac{m^{2}}{4} \theta^{2} \bar{\psi} \square \not \partial \psi-\frac{i m}{12} \theta^{2} \bar{\psi} \square^{2} \psi+\frac{5 m^{4}}{12} \theta^{2} \bar{\psi} \not \partial \psi
\end{aligned}
$$




$$
\begin{aligned}
& +\frac{i m}{6} \theta_{\mu}^{\rho} \theta^{\mu \sigma} \bar{\psi} \partial_{\rho} \partial_{\sigma} \square \psi+\frac{2 m^{4}}{3} \theta^{\rho}{ }_{\mu} \theta^{\mu \sigma} \bar{\psi} \gamma_{\rho} \partial_{\sigma} \psi+\frac{m^{2}}{6} \theta_{\mu}^{\rho} \theta^{\mu \sigma} \bar{\psi} \partial_{\rho} \partial_{\sigma} \not \partial \psi \\
& +\frac{i m^{5}}{4} \theta^{2} \bar{\psi} \psi-\frac{i m^{2}}{4} \tilde{F} \square \tilde{F}-\frac{i m^{2}}{3} \tilde{F}^{\rho \sigma} \square \tilde{F}_{\rho \sigma} \\
& \left.+\frac{i m^{2}}{3} \tilde{F}^{\rho \sigma} \partial_{\sigma} \partial^{\nu} \tilde{F}_{\rho \nu}+\frac{i m^{2}}{6} \tilde{F}^{\rho \sigma} \square \tilde{F}_{\sigma \rho}+\frac{i m^{4}}{2} \tilde{F}_{\rho \sigma} \tilde{F}^{\sigma \rho}\right],
\end{aligned}
$$

Adding (45) and (61) we get the divergent part of the full one-loop corrected effective action for $\mathrm{NC}$ QED:

$$
\begin{aligned}
\Gamma_{1} & =\frac{1}{(4 \pi)^{2} \epsilon} \int d^{4} x\left[4 i \bar{\psi} \not \partial \psi-16 m \bar{\psi} \psi-\frac{2}{3} F_{\mu \nu} F^{\mu \nu}\right. \\
& +\theta^{\alpha \beta}\left(\frac{1}{3} \bar{\psi} \gamma_{\alpha} \partial_{\beta}(\square-i m \not \partial) \psi+\frac{1}{6} \bar{\psi} \sigma_{\alpha \beta} \square(i \not \partial-m) \psi\right. \\
& \left.+m^{2} \bar{\psi} \gamma_{\alpha} \partial_{\beta} \psi+\frac{m^{2}}{2} \bar{\psi} \sigma_{\alpha \beta}(i \not \partial-m) \psi\right) \\
& -\frac{1}{120} \tilde{F}^{\rho \sigma} \square^{2} \tilde{F}_{\sigma \rho}+\frac{1}{30} \tilde{F}^{\rho \sigma} \square^{2} \tilde{F}_{\rho \sigma}-\frac{1}{30} \tilde{F}^{\rho \sigma} \square \partial_{\sigma} \partial^{\nu} \tilde{F}_{\rho \nu} \\
& +\frac{m^{2}}{6} \tilde{F}^{\rho \sigma} \square \tilde{F}_{\rho \sigma}-\frac{m^{2}}{12} \tilde{F}^{\rho \sigma} \square \tilde{F}_{\sigma \rho}-\frac{m^{2}}{6} \tilde{F}^{\rho \mu} \partial_{\mu} \partial^{\nu} \tilde{F}_{\rho \nu}-\frac{m^{4}}{4} \tilde{F}_{\rho \sigma} \tilde{F}^{\sigma \rho} \\
& +\frac{i}{48} \theta^{2} \bar{\psi} \square^{2} \not \partial \psi-\frac{i}{24} \theta^{\alpha \mu} \theta^{\beta}{ }_{\mu} \bar{\psi} \square \not \partial \partial_{\alpha} \partial_{\beta} \psi-\frac{i}{12} \theta^{\alpha \mu} \theta_{\mu}^{\beta} \bar{\psi}^{2} \gamma_{\alpha} \partial_{\beta} \psi \\
& +\frac{m^{2}}{12} \theta^{\alpha \mu} \theta^{b}{ }_{\mu} \bar{\psi} \partial_{\rho} \partial_{\sigma} \square \psi \\
& +\frac{5 i m^{2}}{48} \theta^{2} \bar{\psi} \square \not \partial \psi-\frac{i m^{2}}{24} \theta^{\alpha \mu} \theta_{\mu}^{\beta} \bar{\psi} \not \partial \partial_{\alpha} \partial_{\beta} \psi-\frac{7 i m^{2}}{24} \theta^{\alpha \mu} \theta_{\mu}^{\beta} \bar{\psi}_{\alpha} \partial_{\beta} \square \psi \\
& +\frac{m^{3}}{4} \theta^{\alpha \mu} \theta_{\mu}^{\beta} \bar{\psi} \partial_{\alpha} \partial_{\beta} \psi \\
& +\frac{5 i m^{4}}{24} \theta^{2} \bar{\psi} \not \partial \psi-\frac{i m^{4}}{3} \theta^{\alpha \mu} \theta_{\mu}^{\beta}{ }_{\mu} \bar{\psi} \gamma_{\alpha} \partial_{\beta} \psi \\
& \left.-\frac{m^{5}}{8} \theta^{2} \bar{\psi} \psi\right]+\Gamma_{b} \cdot
\end{aligned}
$$

\section{Conclusions and outlook}

Our goal in this paper was to obtain the divergent part of the one-loop effective action in $\mathrm{NC}$ QED in the second order in the noncommutativity parameter $\theta$ and the same order in the classical fields, $\psi, A^{\mu}$. Thus we obtained the second order corrections to the propagators in the theory and therefore the form of counterterms necessary for renormalization. The method we used is the background field method; the initial point for the perturbative expansion is (29). It is written in such a way that it is easy to sample out the terms contributing to the 2-point, 3-point, 4-point etc. functions. For example, the terms with 3 external fields (corrections to 3 -vertices) are, in the linear order in $\theta$ : $\left(\square^{-1} N_{0}\right)^{k}\left(\square^{-1} N_{1}\right)^{2} \square^{-1} T_{1}$ and $\left(\square^{-1} N_{0}\right)^{k} \square^{-1} N_{1} \square^{-1} T_{2}$; in the quadratic 
order in $\theta$ : $\left(\square^{-1} N_{0}\right)^{k} \square^{-1} N_{1}\left(\square^{-1} T_{1}\right)^{2} . k$ can be determined from the condition that the corresponding integrals are divergent. The corrections to the 4-point functions are given by the terms: $\theta$-linear: $\left(\square^{-1} N_{0}\right)^{k}\left(\square^{-1} N_{1}\right)^{3} \square^{-1} T_{1}$ and $\left(\square^{-1} N_{0}\right)^{k}\left(\square^{-1} N_{1}\right)^{2} \square^{-1} T_{2}$; $\theta$-quadratic: $\left(\square^{-1} N_{0}\right)^{k}\left(\square^{-1} N_{1}\right)^{2}\left(\square^{-1} T_{1}\right)^{2}$ and $\left(\square^{-1} N_{0}\right)^{k}\left(\square^{-1} T_{2}\right)^{2}$.

Because of the length of calculations we confined ourselves in this paper to the corrections to the 2-point functions. However, in some cases it is not difficult to extract other results. E.g., the divergent part of the 4-fermion vertex, in the linear order in $\theta$, is easily found to be

$$
S_{4 \psi}=-\frac{1}{(4 \pi)^{2} \epsilon} \frac{1}{2} \theta^{\rho \sigma} \Delta_{\sigma \rho \mu}^{\alpha \beta \gamma} \int d^{4} x \bar{\psi} \gamma^{\mu} \psi \bar{\psi} \gamma_{\alpha} \gamma_{\beta} \gamma_{\gamma} \psi
$$

in agreement with [15].

As we have already pointed out, our main motive was to check the renormalizability of $\theta$-expanded NC QED in the first and second order in $\theta$ and the possibilities of generalization to all orders. This was done for the pure $\mathrm{NC} \mathrm{U}(1)$ in [15]. The trick which was used is that the SW map does not fix the fields in the $\theta$-expansion fully, but allows for their redefinitions. If the fields are expanded as (symbolically written)

$$
\hat{A}_{\mu}=\sum \theta^{n} A_{\mu}^{(n)}, \quad \hat{\psi}=\sum \theta^{n} \psi^{(n)},
$$

the allowed redefinitions are of the form

$$
\begin{aligned}
& A_{\mu}^{(n)^{\prime}}=A_{\mu}^{(n)}+\mathbf{A}_{\mu}^{(n)}, \\
& \psi_{\mu}^{(n)^{\prime}}=\psi_{\mu}^{(n)}+\Psi_{\mu}^{(n)},
\end{aligned}
$$

where $\mathbf{A}_{\mu}^{(n)}, \Psi_{\mu}^{(n)}$ are gauge covariant expressions of appropriate dimension with exactly $n$ factors of $\theta$. These field redefinitions produce in the action extra terms of the following forms [15]:

$$
\begin{gathered}
\Delta S_{A}=\int d^{4} x\left(D_{\nu} F^{\mu \nu}\right) \mathbf{A}_{\nu}^{(n)} \\
\Delta S_{\psi}=\int d^{4} x\left[\bar{\psi}(i D-m) \mathbf{\Psi}^{(n)}+\overline{\mathbf{\Psi}}^{(n)}(i D-m) \psi\right] .
\end{gathered}
$$

So if the renormalizability of the theory can be achieved by the field redefinitions, all counterterms have to be of the types (67-68).

First, it is easy to see that in the purely bosonic case the action (44) is of the type (67). Let us discuss what happens when the fermions are present. All bosonic corrections are of the $\theta^{2}$-order, and all are of the allowed type except for the term $-\frac{1}{(4 \pi)^{2} \epsilon} \frac{m^{4}}{4} \tilde{F}_{\rho \sigma} \tilde{F}^{\sigma \rho}$. The fermionic $\theta$-linear correction is

$$
\begin{aligned}
& \frac{1}{(4 \pi)^{2} \epsilon} \theta^{\alpha \beta}\left[\frac{1}{3} \bar{\psi} \gamma_{\alpha} \partial_{\beta}(\square-i m \not \partial) \psi+\frac{1}{6} \bar{\psi} \sigma_{\alpha \beta} \square(i \not \partial-m) \psi\right. \\
+ & \left.m^{2} \bar{\psi} \gamma_{\alpha} \partial_{\beta} \psi+\frac{m^{2}}{2} \bar{\psi} \sigma_{\alpha \beta}(i \not \partial-m) \psi\right] .
\end{aligned}
$$


One can check that the pieces $\frac{1}{3} \bar{\psi} \gamma_{\alpha} \partial_{\beta} \square \psi+\frac{1}{6} \bar{\psi} \sigma_{\alpha \beta} \square(i \not \partial-m) \psi$ and $m^{2} \bar{\psi} \gamma_{\alpha} \partial_{\beta} \psi+$ $\frac{m^{2}}{2} \bar{\psi} \sigma_{\alpha \beta}(i \not \partial-m) \psi$ are allowed by field redefinitions, leaving the term $-\frac{i m}{3} \bar{\psi} \gamma_{\rho} \partial_{\sigma} \psi$ excessive. Thus we see that, indeed, renormalizability cannot be achieved in the massive case, $m \neq 0$, as there are two terms (one bosonic and one fermionic) which prevent it. Both terms are proportional to $m$.

One observes further that for $m=0$ also the $\theta^{2}$ fermionic contribution fits into the field redefinition scheme. Namely this contribution reads

$$
\frac{1}{(4 \pi)^{2} \epsilon}\left[\frac{i}{48} \theta^{2} \bar{\psi} \square^{2} \not \partial \psi-\frac{i}{24} \theta^{\alpha \mu} \theta_{\mu}^{\beta} \bar{\psi} \square \not \partial \partial_{\alpha} \partial_{\beta} \psi-\frac{i}{12} \theta^{\alpha \mu} \theta_{\mu}^{\beta} \bar{\psi}^{2} \gamma_{\alpha} \partial_{\beta} \psi\right]
$$

and it is of the form $(68)$ for $\boldsymbol{\Psi}^{(2)}$ given by

$$
\Psi^{(2)}=\frac{1}{(4 \pi)^{2} \epsilon}\left[\frac{1}{96} \theta^{2} \square^{2} \psi-\frac{1}{48} \theta^{\alpha \mu} \theta_{\mu}^{\beta} \square \partial_{\alpha} \partial_{\beta} \psi-\frac{1}{24} \theta^{\alpha \mu} \theta_{\mu}^{\beta} \square \not \partial \gamma_{\alpha} \partial_{\beta} \psi\right] .
$$

For completeness, let us rewrite the effective action in the massless case:

$$
\begin{aligned}
\Gamma_{1, m=0} & =\frac{1}{(4 \pi)^{2} \epsilon} \int d^{4} x\left[4 i \bar{\psi} \not \partial-\frac{2}{3} F_{\mu \nu} F^{\mu \nu}\right. \\
& +\theta^{\alpha \beta}\left(\frac{1}{3} \bar{\psi} \gamma_{\alpha} \partial_{\beta} \square \psi+\frac{1}{6} \bar{\psi} \sigma_{\alpha \beta} \square i \not \partial \psi\right) \\
& -\frac{1}{120} \tilde{F}^{\rho \sigma} \square^{2} \tilde{F}_{\sigma \rho}+\frac{17}{60} \tilde{F}^{\rho \sigma} \square^{2} \tilde{F}_{\rho \sigma}+\frac{1}{20} F^{\rho \sigma} \square \tilde{\partial}_{\mu} \tilde{\partial}^{\mu} F_{\rho \sigma}-\frac{1}{16} \theta^{2} F^{\rho \sigma} \square^{2} F_{\rho \sigma} \\
& \left.+\frac{i}{48} \theta^{2} \bar{\psi} \square^{2} \not \partial \psi-\frac{i}{24} \theta^{\alpha \mu} \theta_{\mu}^{\beta} \bar{\psi} \square \not \partial \partial_{\alpha} \partial_{\beta} \psi-\frac{i}{12} \theta^{\alpha \mu} \theta_{\mu}^{\beta} \bar{\psi}^{2} \gamma_{\alpha} \partial_{\beta} \psi\right] .
\end{aligned}
$$

To summarize: our calculation inforces the conclusions of 19 (to the $\theta^{2}$-order): the propagators in NC QED can be renormalized by the field redefinitions if fermions are massless; for massive fermions this is not possible. Unfortunately, as also stressed in 19], the full renormalizability is spoiled by the term (63) arousing in the correction of 4 -fermions vertex. This correction is not of the form (68) (has no derivatives), and therefore cannot be obtained by field redefinitions.

One may expect that the $\mathrm{NC}$ versions of nonabelian gauge theories have different properties from $\mathrm{NC} \mathrm{U}(1)$. On the other hand, the similar approach to the problem of renormalizability seems to be viable, at least for $S U(2)$ Yang-Mills theory. This will be the subject of the fortcoming publications.

\section{Appendix}

Using Bianchi identities we obtained the following expressions:

$$
\partial_{\rho} \partial_{\sigma} \tilde{F}^{\rho \sigma}=-\frac{1}{2} \square \tilde{F},
$$




$$
\begin{gathered}
\tilde{\partial}^{\alpha} F^{\rho \sigma} \tilde{\partial}_{\alpha} F_{\rho \sigma}=-2 \tilde{F}^{\mu \nu} \square \tilde{F}_{\mu \nu}+2 \tilde{F}^{\alpha \sigma} \partial_{\rho} \partial_{\sigma} \tilde{F}_{\alpha}{ }^{\rho} \\
\tilde{F}^{\mu \rho} \partial^{\nu} \partial_{\rho} \tilde{F}_{\nu \mu}=\tilde{F}^{\mu \rho} \partial_{\mu} \partial^{\nu} \tilde{F}_{\nu \rho}=-\frac{1}{2} \tilde{F} \partial_{\mu} \partial_{\nu} \tilde{F}^{\mu \nu} \\
\tilde{F}^{\mu \rho} \partial^{\nu} \partial_{\mu} \tilde{F}_{\rho \nu}=\frac{1}{2} \tilde{F}_{\mu \nu} \square \tilde{F}^{\nu \mu}, \\
F^{\alpha \nu} \partial_{\alpha} \partial^{\mu} F_{\mu \nu}=\frac{1}{2} F^{\mu \nu} \square F_{\mu \nu} .
\end{gathered}
$$

Identities including $\Delta_{\sigma \rho \mu}^{\alpha \beta \gamma}$ :

$$
\begin{gathered}
\Delta_{\sigma \rho \mu}^{\alpha \beta \gamma} \delta_{\alpha}^{\mu}=2\left(\delta_{\sigma}^{\beta} \delta_{\rho}^{\gamma}-\delta_{\sigma}^{\gamma} \delta_{\rho}^{\beta}\right) \\
\theta^{\rho \sigma} \theta^{r s} \Delta_{\sigma \rho \mu}^{\alpha \beta \gamma} \Delta_{s r m}^{a b c} \delta_{\alpha}^{\mu} \delta_{a}^{m}=16 \theta^{\gamma \beta} \theta^{c b} \\
\theta^{\rho \sigma} \theta^{r s} \Delta_{\sigma \rho \mu}^{\alpha \beta \gamma} \Delta_{s r m}^{a b c} \delta_{\alpha}^{m} \delta_{a}^{\mu}=8\left(\theta^{b \beta} \theta^{c \gamma}-\theta^{c \beta} \theta^{b \gamma}\right) \\
\theta^{\rho \sigma} \theta^{r s} \Delta_{\sigma \rho \mu}^{\alpha \beta \gamma} \Delta_{s r m}^{a b c} g_{b \beta} g_{c \gamma} g^{m \mu}=-8 \theta^{\alpha}{ }_{\rho} \theta^{\rho \alpha}+4 \theta^{2} g^{a \alpha}
\end{gathered}
$$

\section{References}

[1] J. Madore, S. Schraml, P. Schupp and J. Wess, Eur. Phys. Jour. C16 (2000) 161

[2] B. Jurčo, L. Moller, S. Schraml, P. Schupp and J. Wess, Eur. Phys. J. C21 (2001) 383

[3] J. Madore, An Introduction to Noncommutative Differential Geometry and its Physical Applications, Camb. Univ. Press, Cambridge, 1999

[4] M. Hayakawa, "Perturbative analysis on infrared and ultraviolet aspects on noncommutative QED on $R^{4}$, preprint hep-th/9912167

[5] L. Bonora, M. Schnabl, M. M. Sheikh-Jabbari and A. Tomasiello, Nucl. Phys. B589 (2000) 461

[6] M. Chaichian, P. Prešnajder, M. M. Sheikh-Jabbari and A. Tureanu, Phys. Lett. B 526 (2002) 132

[7] N. Seiberg and E. Witten, JHEP 9909, 032 (1999)

[8] T. Krajewski and R. Wulkenhaar, Int. J. Mod.Phys. A 15 (1999) 1011

[9] S. Minwalla, M. Raamsdonk and N. Seiberg, JHEP 0002, (2000) 020

[10] A. Matusis, L. Susskind and N. Toumbas, JHEP 0012 (2000) 002 
[11] R. J. Szabo, "Quantum field theory on noncommutative spaces", preprint hep-th/0109162

[12] M. R. Douglas and N. A. Nekrasov, Rev. Mod. Phys. 73 (2002) 977

[13] L. Griguolo and M. Pietroni, JHEP 0105 (2001) 032

[14] T. Asakawa and I. Kishimoto, "Comments on Gauge Equivalencein Noncommutative Theory", preprint hep-th/9909139

[15] A. A. Bichl, J.M. Grimstrup, H. Grosse, L. Popp, M. Schweeda and R. Wulkenhar, JHEP 0106 (2001) 013

[16] X. Calmet, B. Jurčo, P. Schupp, J. Wess and M. Wohlgennant, hep$\mathrm{ph} / 0111115$

[17] A. A. Bichl, J.M. Grimstrup, L. Popp, M. Schweeda and R. Wulkenhar, Int. J. Mod. Phys. A17 (2002) 2219

[18] A. A. Bichl, J.M. Grimstrup, L. Popp, M. Schweeda and R. Wulkenhar, "Deformed QED via Seiberg-Witten Map", preprint hep-th/0102103

[19] R. Wulkenhaar, JHEP 0203 (2002) 024

[20] A. Miković and V. Radovanović, Class. Quant. Grav. 15 (1998) 827

[21] A. Miković and V. Radovanović, Nucl. Phys. B 504 (1997) 511

[22] A. G. Barvinsky and G. A. Vilkovisky , Phys. Rep. 119 (1985) 1 